\title{
Erratum to: The vision for MUSE4Music
}

\section{Applying the MUSE method in musicology}

\author{
Johanna Barzen ${ }^{1}$. Uwe Breitenbücher ${ }^{1}$ - Linus Eusterbrock ${ }^{2}$. \\ Michael Falkenthal $^{1}$ - Frank Hentschel ${ }^{2}$ - Frank Leymann ${ }^{1}$
}

Published online: 13 December 2016

(C) Springer-Verlag Berlin Heidelberg 2016

\section{Erratum to: Comput Sci Res Dev DOI 10.1007/s00450-016-0336-1}

Some author photos and biographies were interchanged by mistake. The author photos and biographies are correctly placed here.

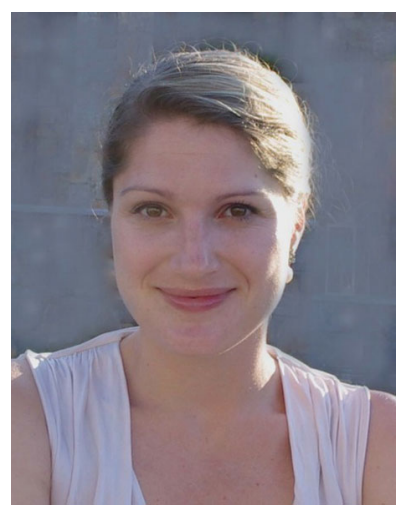

Johanna Barzen studied media science, musicology and phonetics at the University of Cologne and gained first practical experience while working for some major television channels like WDR and RTL. Next to this she studied costume design at the ifs (international film school Cologne) and worked in several film productions in the costume department in different roles. Currently she is Ph.D. student at the University of Cologne and research staff member at the Institute of Architecture of Application Systems (IAAS) at the University Stuttgart doing research on vestimentary communication in film.

The online version of the original article can be found under doi:10.1007/s00450-016-0336-1.

Johanna Barzen

Johanna.Barzen@iaas.uni-stuttgart.de

Uwe Breitenbücher

Uwe.Breitenbuecher@iaas.uni-stuttgart.de

Linus Eusterbrock

Linus.Eusterbrock@uni-koeln.de

Michael Falkenthal

Michael.Falkenthal@iaas.uni-stuttgart.de

Frank Hentschel

Frank.Hentschel@uni-koeln.de

Frank Leymann

Frank.Leymann@iaas.uni-stuttgart.de

1 Institute of Architecture of Application Systems, University of Stuttgart, Stuttgart, Germany

2 Musicological Institute, University of Cologne, Cologne, Germany

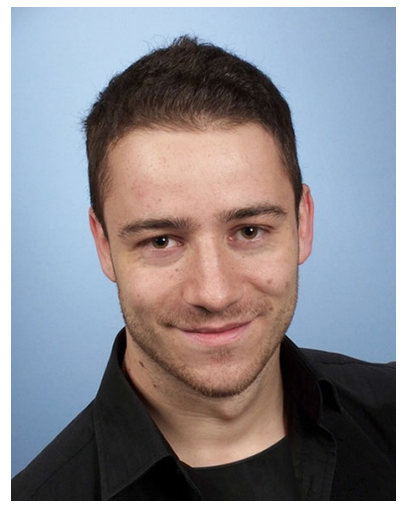

tems and microservices.
Uwe Breitenbücher is a research staff member and postdoc at the Institute of Architecture of Application Systems (IAAS) at the University of Stuttgart, Germany. His research vision is to improve cloud application provisioning and application management by automating the application of management patterns. Uwe was part of the CloudCycle project, in which the OpenTOSCA Ecosystem was developed. His current research interests include cyber-physical sys- 


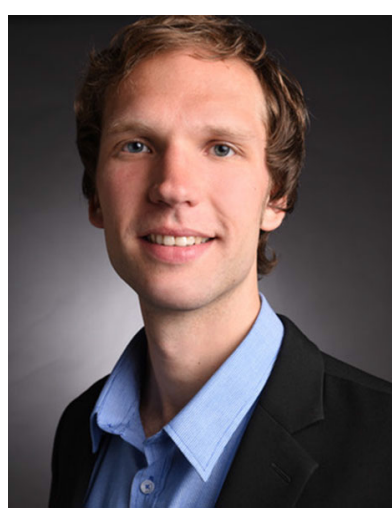

Linus Eusterbrock studied musicology and philosophy at the University of Cologne and at the University Paris-Sorbonne. He worked at the Philharmony in Luxemburg and as a music teacher. Currently, he is research assistant at the Musicological Institute of the University of Cologne, working on historical musicology and Digital Humanities.

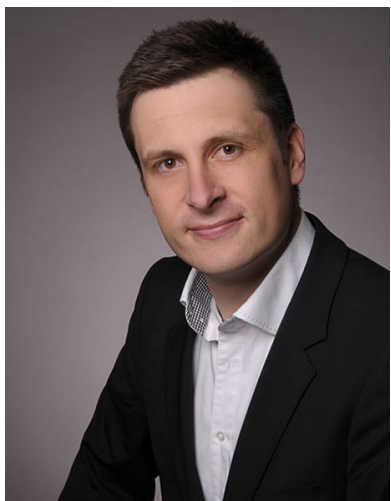

Michael Falkenthal is a research staff member and Ph.D. student at the Institute of Architecture of Application Systems (IAAS) at the University of Stuttgart, Germany. He studied business information technology at the Universities of Applied Sciences in Esslingen and Reutlingen focussing on business process management, services computing and enterprise architecture management. Michael gained experience in several IT transformation and migration projects of small-, medium- and big-sized companies. His current research interests are fundamentals on pattern language theory as well as cloud computing.

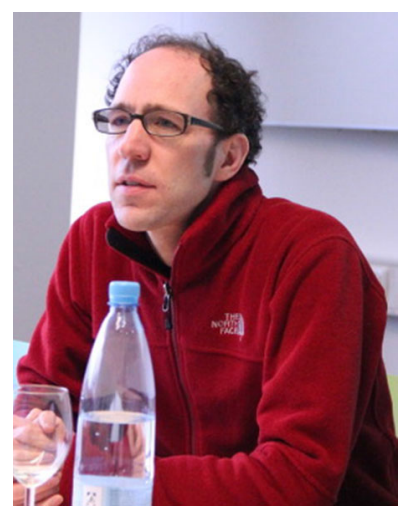

Frank Hentschel is a full professor of musicology at the Musicological Institute at Cologne University. His research fields include medieval music theory, music from 18th to the 19th centuries with a special focus on the social and political functions of music as well as film music. He has published 6 monographs with leading publishing houses and several articles in peer reviewed journals.

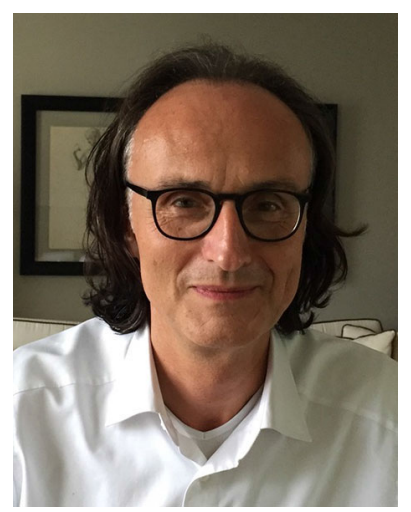

Frank Leymann is a full professor of computer science and director of the Institute of Architecture of Application Systems (IAAS) at the University of Stuttgart, Germany. His research interests include service-oriented architectures and associated middleware, workflow- and business process management, cloud computing and associated systems management aspects, and patterns. Frank is co-author of more than 300 peer-reviewed papers,more than 40 patents, and several industry standards. He is on the Palsberg list of Computer Scientists with highest h-index. 